\title{
CONGENITAL DEFICIENCY OF THE TIBIA
}

\author{
A. KALAMCHI, R. V. DAWE
}

From the Alfred I. duPont Institute, Wilmington, Delaware

\begin{abstract}
The late results of treatment of 24 legs in 21 children with congenital deficiency of the tibia are presented. A new classification is proposed which correlates well with recommendations for treatment and with the final functional result.

Three types of deficiency were recognised: Type I, total absence of the tibia; Type II, distal absence; Type III, distal deficiency with tibiofibular diastasis. The early radiographic appearances, the functional status of the quadriceps and the severity of flexion contracture of the knee were important factors in the selection of the operations likely to give the best function.
\end{abstract}

Congenital deficiency of the tibia, including tibial hemimelia, aplasia and dysplasia, is very rare. It needs to be distinguished from the commoner congenital deficiency of the fibula, which includes fibular hypoplasia and hemimelia. Partial or total absence of the fibula is usually associated with hypoplasia and angulation of the tibia, with the foot in valgus and with the more pronounced deficiency on the lateral side of the limb (Frantz and O'Rahilly 1961; Hootnick et al. 1977; Achterman and Kalamchi 1979).

In congenital deficiency of the tibia, the fibula is usually intact but there is aplasia or marked dysplasia of the tibia. Children with this abnormality are usually born with marked shortening and bowing of the involved leg (Aitken 1971; Jones, Barnes and Lloyd-Roberts 1978). Flexion contracture of the knee and a skin dimple overlying the proximal tibial region are commonly present. The foot is quite rigid in varus and supination, and points inward towards the perineum. There is marked shortening of the first metatarsal, which is frequently associated with other medial ray defects (Fig. 1).

The incidence of congenital deficiency of the tibia in the United States is approximately one per million live births (Brown 1971), and only about 250 cases have been reported in the world literature. Myers (1905-6, 1910-11), first discussed the condition in the North American literature, quoting the first report by Otto in the German literature in 1841 and Albert's 1877 description of treatment by transfer of the fibula beneath the femur to serve as a weight-bearing strut. Myers advised the creation of a functional fibulofemoral arthroplasty; this operation was

A. Kalamchi, MD, Assistant Professor of Orthopaedics and Pediatrics, University Hospitals of Cleveland and Case Western Reserve University School of Medicine

2074 Abington Road. Cleveland, Ohio 44106, USA.

R. V. Dawe, MD, Staff Orthopedist

Bridgeport Hospital, Bridgeport, Connecticut 06430, USA.

Requests for reprints should be sent to Dr A. Kalamchi.

(C) 1985 British Editorial Society of Bone and Joint Surgery

$0301-620 X / 85 / 4131 \$ 2.00$

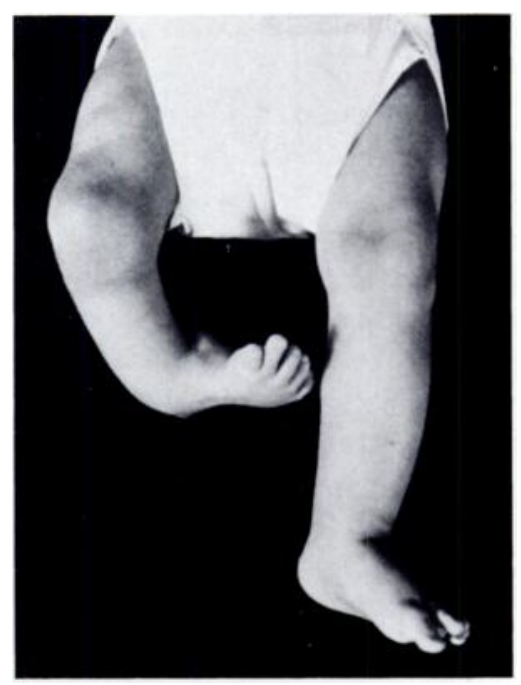

Fig. 1

The typical appearance of a child with congenital tibial deficiency. There is flexion contracture of the knee and medial deviation of a deformed foot. The distal fibula is prominent.

re-introduced and popularised by Brown (1965). Frantz and O'Rahilly (1961) were the first to classify lower limb deficiencies, and Jones et al. (1978) devised a classification of congenital deficiency of the tibia based upon the initial radiographic appearance.

This paper presents our experience in treating 21 children with congenital deficiency of the tibia, and describes our classification of the condition, which is modified from that of Jones et al. (1978). We outline the importance of this classification of the type and degree of deformity, which allows the selection of appropriate treatment at an early stage in the care of the child.

\section{MATERIALS AND METHODS}

The 21 children reported in this series were seen at the Alfred I. duPont Institute between 1950 and 1980. The average age when first seen was six months. There were 9 


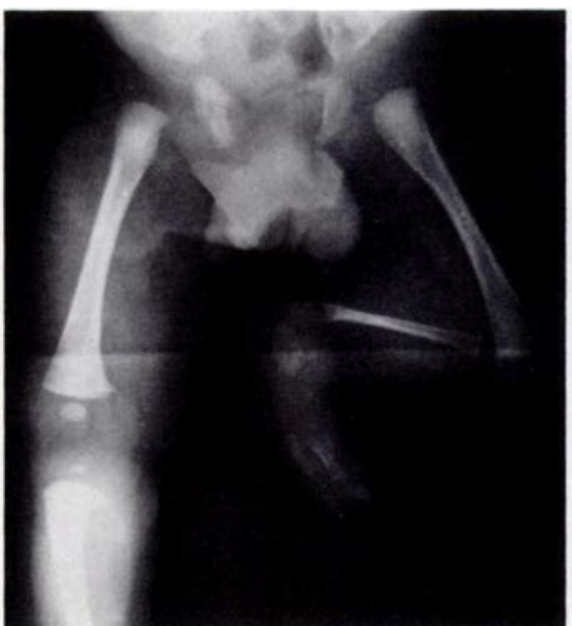

Fig. 2

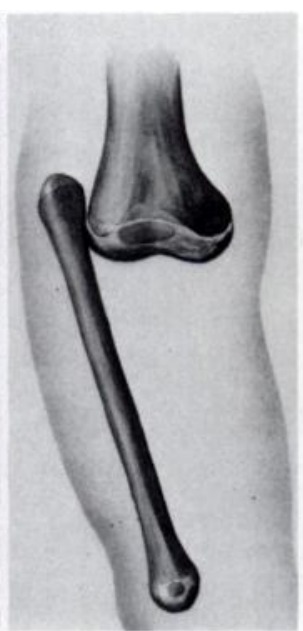

Fig. 3

Radiograph and diagram of Type I deformity demonstrating total absence of the tibia. The radiograph also shows the foot deformity with missing medial rays. and the absence of the distal femoral epiphysis.

boys and 12 girls: three children had congenital deficiency of both tibiae. Of the 24 involved limbs, 17 were on the right and 7 on the left. Complete sets of radiographs were available for all children.

Thorough evaluation of the initial films and assessment of the changes with growth enabled us to classify these patients into three types with differing degrees of tibial deficiency. These types were considered in conjunction with their clinical data and were further characterised. The functional results after treatment were then assessed, allowing us to present guidelines for early management based on the type of deficiency.

\section{CLASSIFICATION}

Three types of deficiency, based on clinical and radiographic findings, were distinguished.

Type I (total absence of the tibia). Of the 24 limbs, 11 had Type I deficiency. The children in this group were born with marked flexion contracture of the knee, variable rotation of the leg, and marked inversion and adduction of the foot, occasionally with deficiency of the medial rays (Fig. 1). Clinically, the knee flexion contracture was over 45 and there was no active contraction of the quadriceps. Radiographs showed total absence of the tibia (Figs 2 and 3). Proximal migration or dislocation of the head of the fibula and marked hypoplasia of the distal femur were also commonly seen. The hypoplastic features of the femur included reduction in the width of the distal metaphysis and marked retardation of the ossification of the distal epiphysis (Jones et al. 1978).

Type II (distal tibial aplasia). The proximal tibia was present in all 10 limbs placed in this group, and formed a relatively normal articulation with the femur. Active quadriceps contraction could usually be elicited. Flexion contracture of the knee was between 25 and $45^{\circ}$, correlating well with the degree of development of the proximal tibia. The initial radiographs showed the proximal

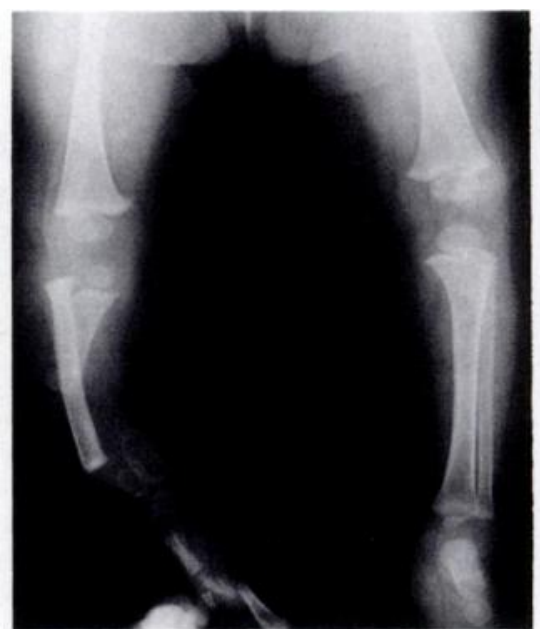

Fig. 4

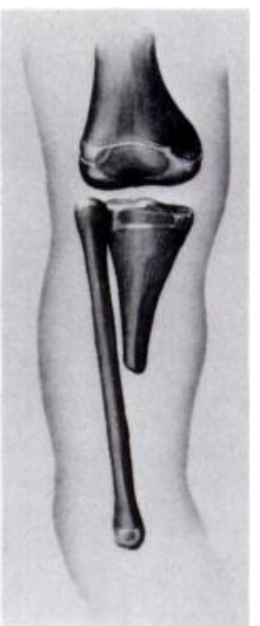

Fig. 5
Radiograph and diagram of Type II deficiency. The proximal tibia is present and the knee is well preserved. There is less proximal migration of the fibula, but the foot is displaced.

tibia in a normal relationship with the distal femur (Figs 4 and 5). If the proximal tibial fragment was small and cartilaginous, it could not be seen on early films; but its presence as an anlage was indicated by the normal development of the distal femur, and in particular by the normal width of the femoral metaphysis and normal ossification of the epiphysis (Figs 6 and 7). Proximal migration of the fibula was not usually as severe as that seen in Type I deficiency.

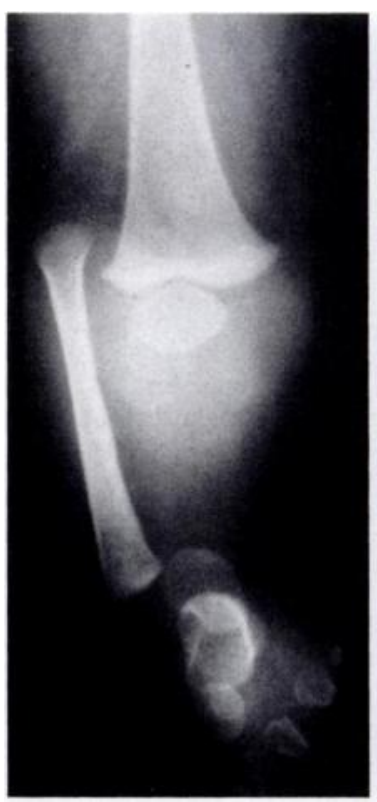

Fig. 6

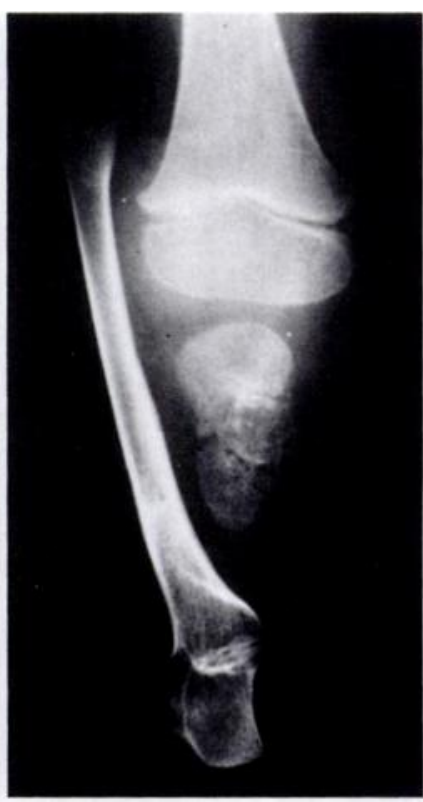

Fig. 7
Another example of Type II deficiency. Figure 6-The tibia is not apparent on the first radiograph though its presence should be deduced from the normal development of the distal femoral epiphysis. Clinical indications of its presence were the stability and the mild degree of flexion contracture of the knee. Figure 7-Two years later the proximal tibia has ossified, and a Boyd amputation has been done. 
Type III (dysplasia of the distal tibia with diastasis of the tibiofibular syndesmosis). The three limbs with Type III tibial deficiency had normal knee joints with a welldeveloped quadriceps mechanism. The degree of diastasis at the ankle varied, but the talus had subluxated proximally, there was prominence of the distal fibula, and the foot was tilted into a varus position. The distal tibia showed hypoplasia and shortening (Fig. 8).

Associated anomalies. Children suffering from tibial deficiency are reported to have a high incidence of associated congenital anomalies (Aitken 1971; Pashayan et al. 1971; Clark 1975; Jones et al. 1978). Two-thirds of the children in our series had such anomalies, of which the most frequent were of the hand $(43 \%)$, the male gonads $(43 \%)$, the other foot $(39 \%)$ and the ipsilateral femur $(29 \%)$. Less frequent anomalies included herniae $(19 \%)$, congenital heart disease $(14 \%)$, learning disabilities $(14 \%)$ and scoliosis $(9.5 \%)$.

The most frequent hand anomaly was lobster-claw deformity in three children, while anomalies of the thumb. including bifid thumb, syndactyly and absence, also were seen in three children. Of the anomalies of the male gonads, three were cryptorchidism and one was a varicocele. Foot anomalies included severe equinovarus deformity in four patients. Involvement of the femur was most commonly by hypoplasia with mild shortening: two of the seven children with this anomaly had radiographic irregularity of the capital femoral epiphysis similar to that seen in Perthes' disease; however, these patients had no symptoms and showed no evidence of subluxation of the hip.

\section{TREATMENT AND RESULTS}

Type I deficiency. Operations on this group of 11 children included three fibular transfers, three knee disarticulations, two femorofibular fusions, and one early aboveknee amputation. The three children who had transfer of the fibula by the classic Brown technique made poor progress because persistent flexion contracture of the knee, with very limited movement and no active quadriceps function, made prosthetic fitting awkward and cosmetically unacceptable. All three children later needed subsequent disarticulation at the knee and now have excellent function with the type of prostheses used for throughknee amputation.

The three children who had early knee disarticulation all have an excellent result. They began walking early with pylons and now use above-knee prostheses. The two children who had femorofibular fusion have good function with the type of prosthesis used for knee disarticulation. Femorofibular fusion helps to lengthen the femoral segment and improves the lever arm of the limb. The one child who had an above-knee amputation has had difficulty with the stump, where bony overgrowth has caused skin problems. There are also difficulties with the control of rotation of the prosthesis on a short proximal thigh segment.

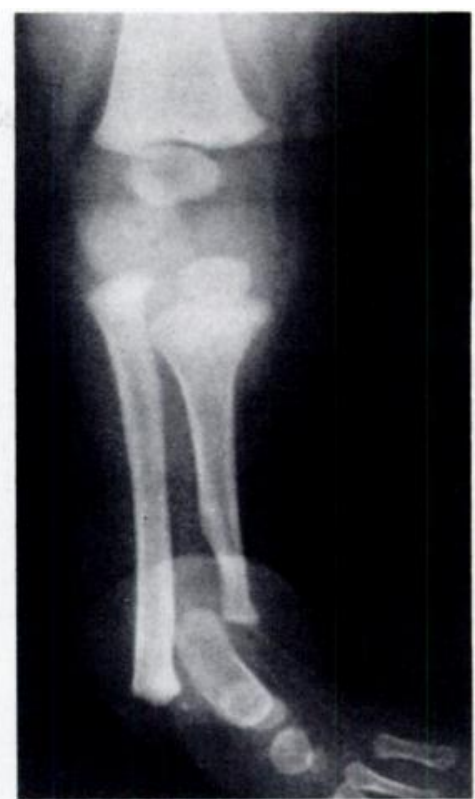

Fig. 8

Radiograph of a child with Type III deformity showing hypoplasia of the distal tibia and diastasis of the tibiofibular syndesmosis.

Type II deficiency. Three operations for transfer of the fibula by a modified Brown procedure, seven tibiofibular synostoses, and two below-knee amputations were performed for this group of 10 patients. Of the seven tibiofibular synostoses, five also had a modified Boyd amputation, in which the os calcis is fused to the distal fibula.

All three children treated by fibular transfer had excellent results and have good function with below-knee prostheses. The modified Brown procedure had stabilised the fibula within the anlage of the proximal tibia and the preservation of growth at the distal fibula had improved the longitudinal growth of the leg. In these children, the presence of the proximal tibia, even though it was not normal, helped to create a fairly stable knee. Active quadriceps function maintained an adequate range of knee movement, and at review there was $20^{\circ}$ to $30^{\circ}$ of flexion contracture at the knee with active flexion of $70^{\circ}$ to $90^{\circ}$ from this position.

The five children who had tibiofibular synostosis and Boyd amputation have good function with belowknee prostheses. The two synostoses without any foot amputation were performed on a boy with bilateral involvement. His feet were preserved by reconstructive surgery and he now has independent function without a prosthesis.

Of the two children who had below-knee amputation, one has a fair result. The other has problems with the skin and the length of the stump, and needs a prosthesis with a thigh lacer. This result is considered to be poor. 
Type III deficiency. Two children in this group had calcaneofibular fusion with a Boyd amputation. These children have excellent function, using below-knee prostheses with supracondylar straps. The third patient walks without a support. though he has some instability at the ankle. and a synostosis is planned for a later date.

\section{CONCLUSIONS}

The initial radiographs of children with congenital tibial deficiency can indicate the presence or absence of a tibial anlage as well as the status of the distal femoral metaphysis and epiphysis. The careful evaluation of these films may be the best guide to the functional integrity of the knee. The presence of some proximal tibia and therefore of some knee joint structure correlates with some function of the quadriceps mechanism.

At the first consultation, a distinction should be made between the various degrees of tibial deficiency, because it is important for these children and their parents that plans for treatment are made early. Our classification into three types of deficiency is simple and functional, and helps the surgeon to select the most appropriate management.

Patients with Type I deficiency should have early disarticulation of the knee, which will enable early walking with a pylon type of prosthesis. Reconstructive procedures aimed at salvaging the knee did not yield good results in this group. The lack of any proximal tibia or of active quadriceps function results in persistence of knee contracture and failure to gain a functional range of movement. Rarely, if the femur is markedly hypoplastic femorofibular fusion can be considered. This will add length to the stump, improving the lever arm and the fitting of a prosthesis. For Type I patients, we consider that above-knee amputation is contra-indicated, primarily because of the bony overgrowth which may follow the operation in childhood.

For patients with Type II deficiency, the ideal treatment is tibiofibular fusion to obtain stability at the knee. This can be done early or at a later stage. If done early, the proximal fibula is embedded in the cartilaginous anlage of the tibia by a modified Brown procedure. Later in life a classic side-to-side tibiofibular synostosis provides stability. These children usually obtain excellent function of the knee, and it is therefore very important that this type of deficiency is recognised and that the knee joints are preserved. Because there is usually marked leg-length discrepancy or abnormality of the foot, or both, these children also benefit from partial amputation of the foot. We prefer to use a modified Boyd amputation, implanting the distal fibula within the body of the os calcis. This helps to maintain stability and, by preserving the distal growth plate, improves longitudinal growth; it also provides an excellent endbearing stump. We feel strongly that below-knee amputation should be avoided in these patients since it may lead to overwhelming difficulties with bony overgrowth and recurrent skin damage at the end of the stump.

For patients with Type III tibial deficiency, the ideal procedure is calcaneofibular fusion, aimed at stabilising the hindfoot and so improving foot function. If this is not possible, talectomy and a modified Boyd amputation are indicated. For marked instability at the tibiofibular diastasis an operation for synostosis is recommended.

\section{REFERENCES}

Achterman CA, Kalamchi A. Congenital deficiency of the fibula. J Bone Joint Surg [Br] 1979:61-B: 133-7.

Aitken GW. Tibial hemimelia. In: Aitken GT. Selected lower-limb anomalies: surgical and prosthetics management. Washington, DC: National Academy of Sciences, 1971:1-19.

Brown FW. The Brown operation for total hemimelia tibia. In: Aitken GT. Selected lower-limb anomalies: surgical and prosthetics management. Washington, DC: National Academy of Sciences, 1971:21-8.

Brown FW. Construction of a knee joint in congenital total absence of the tibia (paraxial hemimelia tibia): a preliminary report. $J$ Bone Joint Surg $[\mathrm{Am}]$ 1965;47-A:695-704.

Clark MW. Autosomal dominant inheritance of tibial meromelia: report of a kindred. $J$ Bone Joint Surg $[\mathrm{Am}]$ 1975;57-A :262-4.

Frantz CH, O'Rahilly R. Congenital skeletal limb deficiencies. $J$ Bone Joint Surg $[\mathrm{Am}] 1961 ; 43-\mathbf{A}: 1202-24$.

Hootnick D, Boyd NA, Fixsen JA, Lloyd-Roberts GC. The natural history and management of congenital short tibia with dysplasia or absence of the fibula: a preliminary report. $J$ Bone Joint Surg [Br] 1977:59-B:267-71.

Jones D, Barnes J, Lloyd-Roberts GC. Congenital aplasia and dysplasia of the tibia with intact fibula: classification and management. J Bone Joint Surg [Br] 1978;60-B:31-9.

Myers TH. Congenital absence of tibia: transplantation of head of fibula, arthroplasty at the ankle joint. Am J Orthop Surg 1905-6; 3:72-85.

Myers TH. Further report on a case of congenital absence of the tibia. Am J Orthop Surg 1910-11:8:398-400.

Pashayan H, Fraser FC, McIntyre JM, Dunbar JS. Bilateral aplasia of the tibia, polydactyly, and absent thumb in father and daughter. J Bone Joint Surg [Br] 1971:53 B:4959. 